Daimon. Revista Internacional de Filosofía, n ${ }^{\circ} 85$ (2022), pp. 177-188

ISSN: 1130-0507 (papel) y 1989-4651 (electrónico)

http://dx.doi.org/10.6018/daimon.410941

Licencia Creative Commons Reconocimiento-NoComercial-SinObraDerivada 3.0 España (texto legal). Se pueden copiar, usar, difundir, transmitir y exponer públicamente, siempre que: i) se cite la autoría y la fuente original de su publicación (revista, editorial y URL de la obra); ii) no se usen para fines comerciales; iii) se mencione la existencia y especificaciones de esta licencia de uso.

(c) (1) (9)

\title{
El problema de la agencia material. Un desafío para el pensamiento posthumanista contemporáneo
}

\section{The problem of material agency. A challenge for contemporary posthumanist thinking}

NICOLÁS LEANDRO FAGIOLI*

\begin{abstract}
Resumen: El presente trabajo se propone reflexionar en torno al vínculo entre la noción de agencia material y el pensamiento posthumanista contemporáneo. Más específicamente nos proponemos cuestionar la necesidad de que una postura filosófica no antropocéntrica deba contener necesariamente una concepción simétrica de agencia. Para esto, en primer lugar, se analizarán los desarrollos de Lambros Malafouris en torno a dicha problemática, con el fin de caracterizar una concepción simétrica de la capacidad agentiva. En contraposición a esto, en segundo término, se explicitará la visión de Tim Ingold al respecto, con el fin de abrir paso a un nuevo enfoque.

Palabras clave: Agencia, Posthumanismo, Ambiente, Materiales, Vida.
\end{abstract}

\begin{abstract}
This paper aims to reflect on the link between the notion of material agency and contemporary post-humanist thinking. More specifically, we propose to question the need that a non-anthropocentric philosophical stance must necessarily contain a symmetrical conception of agency. For this, in the first place, we will analyze the developments of Lambros Malafouris around this problem, in order to characterize a symmetrical conception of the agentive capacity. In contrast to this, in the second place, we will explain Tim Ingold's vision of agency, in order to make way for a new approach.
\end{abstract}

Keywords: Agency, Posthumanism, Environment, Materials, Life

Recibido: 21/01/2020. Aceptado: 03/03/2020.

* Doctorando en Filosofía por la Universidad de Buenos Aires. Profesor de Filosofía del Arte en el Departamento de Artes Musicales y Sonoras de la Universidad Nacional de las Artes.nicofagioli@gmail.com. Sus líneas de investigación actuales son el posthumanismo, los nuevos materialismos, y la problemática de la naturaleza en el materialismo contemporáneo. Sobre esta problemática ha publicado: "Quiasmo, carne y naturaleza. MerleauPonty y el problema de la medialidad" (Revista Areté, Vol. 33, Núm. 1, 2021) y "La naturaleza como entrelazo. Experiencia y ambiente" (Cuadernos de filosofía, 2021, en prensa). 


\section{Introducción}

La agencia ha estado históricamente relacionada con la acción humana. Desde hace algunas décadas, gracias al crecimiento de los nuevos materialismos y de las diferentes corrientes posthumanistas en general $^{1}$, la discusión acerca de la posibilidad de concebir algún tipo de agencia más allá de las fronteras de los vivientes humanos y animales resulta un tópico recurrente. Expandir el alcance de la capacidad agentiva hasta el plano de la materia parecería, en principio, aportar una herramienta fundamental para el descentramiento del existente humano de su lugar privilegiado con respecto al resto de los entes. Si la materia se ha considerado mayormente como un polo pasivo a ser determinado, modelado e informado, los nuevos materialismos pretenden repensarlo en términos de actividad y autodeterminación. No obstante, resistiendo a esta tendencia, numerosos autores sostienen que es imposible hablar de agencia sin referir a un cierto nivel de autonomía, premisa que nos obliga a concebir un escenario conformado por dos polos en el que uno de ellos debe necesariamente adoptar un rol activo. ${ }^{2}$ Para esta última postura hablar de agencia remite inevitablemente a un ordenamiento asimétrico de los entes. Dado este contrapunto, la cuestión de la agencia se encuentra anclada en el centro de los debates antropológicos siendo usufructuada para designios contrapuestos.

Más allá de la disputa particular de la agencia, parecería en principio un concepto fundamental en la discusión acerca del vínculo entre lo humano y lo no humano. No quedan dudas de que la comprensión de algún tipo de actividad en aquello que el modelo sujetado al binomio materia-forma considera inerte, pasivo y necesitado de ser informado externamente, resulta de gran interés para el pensamiento postantropocéntrico. Ahora bien, ¿debe el posthumanismo adherir sin miramientos a la teoría de la agencia material? ¿Resulta la simetría agencial una condición necesaria para adoptar una postura posthumanista? ¿Es posible desde el posthumanismo sostener una noción asimétrica de agencia?

En el presente trabajo pretendo introducirme en estas cuestiones con el objetivo de contestar estos interrogantes. Para esto será necesario un cambio de enfoque que nos permita superar ciertas aporías. En miras de este cambio, el camino hacia estos propósitos consta de dos partes: en primer lugar analizaré la concepción de la agencia material de Lambros Malafouris, autor al cual considero como el que más esmero invirtió en argumentar a favor de una simetría agentiva. Tanto él como otros autores, entre los que se destacan Bruno Latour ${ }^{3}$

1 Por posthumanismo entendemos la herencia contemporánea de la tradición de pensamiento postnietzscheana pionera en el descentramiento de lo humano, también denominada antihumanismo y cuya base fundamental es el pensamiento de autores como Michel Foucault, Jacques Derrida o incluso Gilles Deleuze. Sin embargo, se desmarca de este tanto como del humanismo, dado que, como dice Karen Barad: "Posthumanism doesn't presume the separateness of any- 'thing', let alone the alleged spatial, ontological, and epistemological distinction that setshuman apart" $(2007,136)$. En base a esto, el posthumanismo evita sostener al existente humano en el centro de la reflexión ya sea para ponderar sus facultades (humanismo) o para ubicarse en el polo contrario (antihumanismo). El posthumanismo entonces reflexiona en torno a la posibilidad de descentrar al existente humano de su lugar privilegiado con respecto al resto de los entes, tanto ontológica como epistemológicamente, alejándose de una teleología antropocentrada. En base a esto, no involucraremos en el análisis a las corrientes transhumanistas, cuya consideración de lo humano, según consideramos no concuerdan con nuestra concepción del posthumanismo.

2 Véase Di Paolo, E., Barandarian, X. y Rohde, M. (2009), "Defining Agency: Individuality, Normativity, Asymmetry, and Spatio-temporality in Action”, Adaptive Behavior, 17 (5), pp. 367-386.

3 Latour, B. (1994) “On technical mediation. Philosophy, sociology, genealogy”, Common knowledge, 3, pp. 29-64. 
o Arthur Gell ${ }^{4}$, son invocados constantemente en los debates acerca de la centralidad de lo humano y el rol que ocupa la agencia en la discusión. Será a través de Malafouris entonces que en el presente análisis se explicitará la postura que sostiene la posibilidad de pensar dicha categoría en términos materiales, y defender una simetría ontológica en la distribución de la misma. Seguido a este diagnóstico abrevaré en el pensamiento de Tim Ingold. A través de su interpretación de la categoría de ambiente de J.J. Gibson y sus posteriores desarrollos en torno a la noción de materiales, intentaré presentar un nuevo modo de analizar la problemática de la agencia, especialmente cuando se la relaciona de manera cercana con el concepto de intencionalidad. El propósito general reside en explicitar el porqué de la necesidad de un cambio de enfoque con respecto a la agencia material, que integre sus dificultades y, a su vez, realice un aporte al pensamiento posthumanista.

\section{El problema de la agencia material}

El conflicto al cual intentaremos contraponer una propuesta superadora, podría plantearse en los siguientes términos: Si existe la posibilidad de vislumbrar una simetría en cuanto a la capacidad agentiva de los entes, entonces la agencia es un concepto solidario al posthumanismo. Ahora bien, si se llegara a la conclusión contraria, es decir, la imposibilidad de argumentar a favor de dicha simetría ¿es esto necesariamente un obstáculo para la reflexión posthumanista y para el pensamiento materialista en general ${ }^{5}$

Numerosos autores se han ocupado del relativamente nuevo problema filosófico de la agencia material. Consideramos que particularmente dos de ellos resultan ejemplos paradigmáticos del modo en que dicho concepto funciona como una fuente de debilitamiento para el lugar privilegiado que el viviente humano ha construido con respecto a su entorno. En primer término, el pensamiento de Bruno Latour ha sido una punta de lanza para renovar el modo en que concebimos nuestra relación con el mundo circundante. Su modo de abordar la relación entre las intencionalidades humanas respecto a los entes no humanos apunta hacia una concepción simétrica de la agencia. Dicha concepción es en principio metodológica y, más adelante, ontológica. ${ }^{6}$ En segundo lugar, la postura del arqueólogo Lambros Malafouris resulta mucho más radical y enteramente ontológica. Por cuestiones de extensión y por ser

4 Gell, A. (1998), Art and agency. An anthropological theory, Oxford: Clarendon Press.

5 Dadas las múltiples significaciones y la enorme tradición filosófica del concepto de materialismo, nos posicionaremos dentro del marco teórico de un materialismo no-dialéctico y no-histórico, representado principalmente por los denominados nuevos materialismos. Diversos corrientes pueden ser incluidas dentro del materialismo contemporáneo, entre ellas destacamos las siguientes: cuestionamientos al binarismo naturaleza-cultura (P. Descola, B. Latour), estudios acerca de lo posthumano (R. Braidotti, F. Ferrando), el materialismo vibratorio (J. Bennett), el realismo agencial (K. Barad), el realismo especulativo y la ontología orientada a objetos (Q. Meillasoux, G. Harman) y el materialismo posthumano (N. Billi, P. Fleisner, G. Lucero).

6 Pueden detectarse en su pensamiento dos momentos bien claros. Por un lado, una propuesta metodológica que apunta a problematizar la relación del investigador sociológico con los mediadores materiales. Por el otro un progresivo desplazamiento del enfoque a partir del cual puede inferirse una concepción de la agencia que excede el consejo metodológico para incurrir en terreno ontológico. Como vemos, hay una tendencia gradual ascendente hacia una concepción cada vez más equilibrada entre humanos y no humanos con respecto a sus capacidades agentivas. A pesar de que desde Nunca fuimos modernos (1993), con un carácter fuertemente metodológico, se infiltran aseveraciones ontológicas, me centraré en textos posteriores en los que esta última tendencia resulta indiscutible. 
el menos frecuente en la bibliografía específica sobre este tema, desarrollaré sólo esta última postura. Además, la posición de Malafouris con respecto a la no exclusividad humana de la agencia resulta mucho más vehemente y firme, con lo cual, los problemas que a ella le atañen pueden trasladarse también a cualquier otra reflexión más contemplativa y menos impetuosa.

En “At the Potter's Wheel: An Argument for Material Agency" (2008), Malafouris se compromete de un modo directo con el carácter ontológico de la agencia, extendiendo el alcance de la misma a todo tipo de entidad. En principio, no sostiene una distribución democrática de la agencia, al estilo de Latour, sino que la concibe como una propiedad emergente que se manifiesta en el involucramiento material (engagement) en el cual se ve imbricado lo humano y lo no-humano. En palabras del autor: "Si la agencia y la intencionalidad no son propiedades de las cosas, no son propiedades de los humanos tampoco, son propiedades de un involucramiento material, esto es, una zona gris en donde cerebro, cuerpo y cultura se combinan" (2008, 22) ${ }^{7}$. Como afirma Diego Parente "el peso del argumento de Malafouris reside en trasladar la cuestión de la agencia a la pregunta por sus modos de manifestación" $(2016,147)$.

Sumado a este giro de concepción, Malafouris hace explícita la lectura posthumanista de la agencia material, dice el autor: "No podemos suturar la brecha cartesiana entre personas y cosas sin estar dispuestos a compartir una parte sustancial de nuestra eficacia agentiva humana con los medios que hicieron posible en primer lugar el ejercicio de tal eficacia" $(2013,149) .{ }^{8}$ Como vemos, el hecho de entender la cuestión en términos ya no de atribución sino de emergencia constituye en sí mismo una toma de posición con respecto a la problemática posthumanista. La comprensión de la agencia como una propiedad exclusiva del existente humano, según el autor, responde a la capacidad humana de experimentar voluntad consciente y generar un desequilibrio entre la causación mental y la física. Esto es, los humanos poseemos la capacidad de experimentar lo que Malafouris caracteriza como un sentido de agencia, poseemos la habilidad de referir a nosotros mismos como autores de nuestras acciones. Sin embargo, poseer un sentido de agencia no equivale a ser los únicos poseedores de capacidad agentiva, de hecho, no equivale a poseer agencia de ningún tipo. El primer paso, entonces, por medio del cual Malafouris debilita la exclusividad de la capacidad agentiva humana es entendiendo la causalidad como algo aparente y dependiente de un sentido de agencia.

El segundo punto de apoyo fuerte que atacará su argumento es la noción de intencionalidad. Parecería que la agencia, en tanto concepto hermanado con la intencionalidad, deriva de la capacidad humana de anticiparse a las acciones. Si el ser humano puede prever sus actos, no deberían quedar dudas de que esta conciencia garantiza su capacidad agentiva. Se deberá entonces investigar si la naturaleza de la agencia es o no intencional. Para esto, el autor recurre a la caracterización de agencia con perspectiva internalista de John Saerle. En Intentionality: An Essay in the Philosophy of Mind (1983), Saerle concibe a los estados intencionales como proyecciones que apuntan, se enfocan y se prolongan a través de los objetos y representaciones. Los objetos no pueden presentar estados intencionales. Por lo tanto, si la naturaleza de la agencia es intencional, es entonces también una propiedad humana ¿Cómo disociar agencia de intencionalidad? Malafouris recurre a dos estrategias, ambas basadas en el pensamiento del filósofo norteamericano. En la primera propone distinguir dos tipos de estados intencionales: Intenciones previas (PI) e Intenciones en acción

7 La traducción es mía en todos los casos en que se cite este escrito.

8 La traducción es mía. 
(IA). La primera de ellas refiere a la acción premeditada o deliberada en donde la intención de actuar es previa. En este caso existe una representación de las condiciones de satisfacción. La segunda se identifica con la actividad no deliberada, cotidiana, el estado intencional no es distinguible de la acción, aquí más que representación hay una presentación de sus condiciones de satisfacción. En principio, dado que la agencia se relaciona con eventos causales, no parece posible pensarla en PI antes de que encuentre sus condiciones de satisfacción, es decir, cuando se convierta en IA. Por lo tanto, si la agencia y la intencionalidad pueden ser vinculadas, sólo podrá llevarse a cabo esta relación en IA, es decir en el mundo de la práctica actual, no con el pensamiento privado. Aquí, aunque de una manera muy tenue, puede observarse un primer alejamiento de la agencia con respecto a la intencionalidad.

Una segunda estrategia complejiza aún más la cuestión. Las intenciones previas, aquellas que según el autor no tienen vínculo con la agencia, podrían considerarse en algunos casos como la causa de las acciones. Sin embargo, aun separando claramente ambas esferas, la interna de la intención y la externa de la acción, ambas poseen un condicionante. Siempre siguiendo la huella de Saerle, Malafouris suscribe que tanto la intención como la acción están siendo modeladas por un background. Afirma Saerle:

El background no es un conjunto de cosas ni un conjunto de relaciones misteriosas entre nosotros y las cosas, más bien es simplemente un conjunto de habilidades, posturas, asunciones pre-intencionales y presuposiciones, prácticas y hábitos. Y todos ellos, tanto como sabemos, son consumados en los cerebros y cuerpos humanos $(1983,154) .{ }^{9}$

Es decir que el background permite a las intenciones y representaciones tener lugar. Por lo tanto, el engagement o involucramiento material siempre precede a la intencionalidad. El background deviene parte de la mente o lo que puede denominarse estado intencional extendido, las cosas se proyectan sobre mí, tanto como yo me proyecto sobre ellas. La intencionalidad debe ser entendida, entonces, como un fenómeno distribuido, emergente e interactivo más que un estado mental subjetivo. Si la intencionalidad depende del background, entonces no es una propiedad interna de la mente, es por esto que no puede ser un criterio para atribuir agencia de un modo exclusivo a los humanos.

Como vemos, más que disociar a la agencia de la intencionalidad, Malafouris lo que lleva a cabo es un debilitamiento de la concepción de la intencionalidad como perteneciente exclusivamente al sujeto. Si la agencia depende de la intencionalidad, entonces deberá también entenderse en términos de emergencia y no podrá ser una propiedad interna de la mente. Toda acción, en términos agenciales, debe entenderse como una danza sinérgica y simétrica entre la acción humana, las mediaciones productivas y las condiciones singulares materiales. Es en este compuesto entre cerebro, cuerpo y recursos que la agencia se manifiesta a partir de la irreductible tensión de la actividad mediada en el involucramiento material. No hay roles agentivos establecidos, la agencia pertenece al compuesto, y puede ser caracterizada "acorde a aquel componente que en determinado momento tenga la mano arriba en la lucha fenomenológica" (2008, 34). Lo que una entidad es, no tiene importancia, importa en qué deviene y qué papel juega en la red del involucramiento material.

9 La traducción es mía 
Ahora bien, ¿qué problemas conlleva defender una postura de este tipo? Si bien Malafouris, al entender la agencia en términos de emergencia, no arriba a la misma conclusión democrática, en cuanto a la distribución de la misma, al estilo de Latour, sí se inscribe dentro de las concepciones simétricas de la capacidad agentiva. Es decir, en Malafouris, en principio ningún ente posee agencia en sí mismo, sino que todos son capaces de manifestarla de una u otra manera. Sin embargo, a pesar de que esta concepción de la agencia logra sortear ciertas dificultades, a la hora de observar su teoría desde una perspectiva posthumanista se evidencian algunas cuestiones que no pueden ignorarse si pretende sostenerse a la agencia como una auténtica herramienta para desestabilizar la jerarquía del existente humano. En primer lugar, la introducción de la distinción entre agencia y sentido de agencia es utilizada por el autor para remarcar la separación entre la conciencia de llevar a cabo un acto y la capacidad agentiva del agente con respecto al mismo. No obstante, el hecho de poseer un sentido de agencia justamente termina por favorecer la intención contraria, es decir, establece una asimetría de base difícil de sortear. La diferencia cualitativa entre algunas entidades que poseen un sentido de agencia y algunas que no, genera una serie de problemas que requieren una argumentación más detallada. En definitiva, ¿no es ese sentido de agencia la manifestación de todas aquellas características que refuerzan la concepción antropocéntrica de la misma? El mismo Malafouris reconoce la dificultad de sortear este obstáculo: "Es el lado humano el que hace las elecciones vitales y toma las decisiones importantes." $(2008,25)$. No parece posible sostener una concepción simétrica de la agencia que tolere este fuerte grado de normatividad en el "lado humano".

Diferente resulta la cuestión del background, esta noción implica definitivamente una herida grave al privilegio humano de la intencionalidad. A partir de esta categoría es posible arribar a una noción de agencia extendida o andamiada (Sterelny, 2005) que considere a los componentes del entorno del agente como posibilitadores de acción (affordances) (Gibson, 1986). De este modo se consolida una versión "débil" de agencia material deflacionaria de la exclusividad agentiva humana que, a su vez, evita las dificultades que acarrea la defensa de un enfoque enteramente simétrico. Sin embargo, esto no parece conformar a Malafouris, a pesar de reconocer el peso del polo que posee sentido de agencia, su conclusión se inclina más hacia el lado de la simetría y de una concepción "fuerte" de la agencia material en términos de manifestación y emergencia. ${ }^{10}$ Nos encontramos aquí en una aparente aporía: Por un lado, puede adoptarse una versión débil de agencia material, basada en la noción de background, que no satisfaga las necesidades de simetría agencial y que preserve las condiciones de normatividad interna para el lado humano. De tomar este camino, también deberíamos conformarnos con una concepción posthumanista "débil" de la agencia que no logra debilitar el privilegio humano en cuanto a ella. El otro camino es decidirse directamente por la simetría ontológica, habilitando las críticas y volviendo al punto de partida.

Ahora bien, ¿de qué modo podemos evitar esta última opción, dada la dificultad de superar las críticas, y aún inscribir esta postura dentro de un pensamiento materialista

10 Esto no deja de ser una contradicción en el pensamiento del autor. Sus argumentaciones responden casi sin excepción a una estructura binaria que no abandona la polarización humano-no humano. Sumado a esto, las características que se destacan en el costado humano dificultosamente aporten a la construcción de una concepción simétrica de la agencia. 
posthumano? ¿Existe algún modo de sostener un enfoque posthumanista a la vez que una asimetría agencial? De ser así, considero que esta vía debe encontrarse en el intento de concebir algún polo activo de la materia que no dependa necesariamente de resolver a su favor los múltiples inconvenientes que acarrea la cuestión de la agencia material. Si pudiera pensarse algún tipo de actividad en la materia que no implique necesariamente una atribución agentiva, entonces la reflexión posthumanista podrá observar la problemática de la agencia desde otro lugar. La salida, considero, es necesariamente materialista, si se toma la materia como punto de partida, el pensamiento postantropocéntrico encuentra una avenida fluida para una argumentación fuerte que no ceda en su potencia filosófica.

\section{Un mundo de materiales}

Como vemos resulta dificultoso desactivar el modelo hilemórfico, solidario al antropocentrismo, a través del camino de la agencia material. Aunque parece en principio un concepto funcional a un nuevo enfoque, se generan ciertos problemas que nos devuelven a ciertos binomios tradicionales como forma/materia mente/materia o naturaleza/cultura, es decir, a la dialéctica entre un polo pasivo y uno activo. Si retomamos los interrogantes expuestos al principio, la concepción de un polo activo en la materia, más que la búsqueda de un modo de poner de cabeza el modelo dualista, requiere el abandono de estos dualismos, los cuales la noción de agencia replica. Es decir, no se trata de dar vuelta el modelo animando el polo pasivo sino de cambiar el enfoque y disolver el binomio activo/pasivo, desestimando así las eternas discusiones que acarrean los dualismos consecuentes. Resulta menester adoptar un enfoque que evite caer en los dispositivos binarios y que plantee a la materia como punto de partida de la reflexión. Si bien los denominados nuevos materialismos resultan la salida más evidente para desarrollar este enfoque, para el presente trabajo indagaremos en una vía cercana a las discusiones referentes a la Filosofía de la técnica: la teoría de la percepción del antropólogo Tim Ingold. ¿Por qué recurrir a este autor? Porque nos permite encarar las aporías derivadas de la búsqueda de una simetría agencial desde otra perspectiva y a partir de sus tratamientos puede reconsiderarse la conflictiva relación entre la agencia y el enfoque posthumanista. Si bien su pensamiento intenta evitar el enfoque binario, esto no implica un abandono total de todo dualismo. Más bien se trata de una modificación en el abordaje de los mismos y un cambio de enfoque general que logra desestimar ciertas disputas irresolubles.

La noción que nos ocupa no ha sido particularmente un foco de investigación en la literatura de Ingold, sin embargo, en las contadas menciones que el autor hace de ella se evidencia su visión de la misma. En todas se refiere a la atribución de agencia a entidades no humanas en el mismo tono: es asociada a una necesidad animista de insuflar vida a las cosas. Es decir, una suerte de re-encantamiento del mundo. En Bringing things to life (2010) la agencia es caracterizada como "un intento de reanimar un mundo de las cosas muertas o interpretadas inertes deteniendo el flujo de sustancias que le dan vida" $(2010,6)$. Ingold apunta directamente a la relación entre la atribución de agencia y su cercanía con la necesidad de reconocer un impulso vital en las cosas. En "Los materiales contra la materialidad" es más taxativo y claro en su posicionamiento: 
Sofocado por la mano muerta de la materialidad, este mundo sólo puede ser devuelto a la vida en los sueños de los teóricos al conjurar un polvo mental mágico que, salpicado entre sus componentes, se supone que los pondría físicamente en movimiento. Se lo conoce en la literatura como agencia, y grandes expectativas se han depositado en ella $(2013,32)$.

Contra esto argumenta: "Traer las cosas a la vida no consiste en espolvorearlas con agencia, sino en devolverlas a los flujos generativos del mundo de materiales en el que se originaron y en donde continúan subsistiendo." $(2013,33)$. El problema del impulso animista es que la necesidad de insuflar vida a las cosas requiere considerarlas previamente como muertas o inertes. Como vemos, a diferencia de lo que ocurría con Malafouris, se introduce aquí una problemática nueva: el vínculo entre agencia y vida. El problema subyace en que, al parecer, el único modo de concebir algún tipo de actividad en el polo considerado como pasivo es atribuyendo algún tipo de vitalidad en él. ${ }^{11}$ La agencia funciona como el elemento por antonomasia para cumplir con esta pretensión. Debemos interrogarnos entonces cuán legítima es la relación entre agencia y vida y, aún más, si es este el único modo de concebir algún tipo de actividad en el polo considerado como pasivo. ${ }^{12}$

La propuesta de Ingold concibe al existente humano integrado en los procesos de generación y transformación de los materiales. Esto implica alejarse de una visión en términos de sujeto/objeto, binomio que replica la perspectiva metafísica, por una en términos de persona/ambiente. Para esto recurre al pensamiento de J.J. Gibson quien en The ecological approach to visual perception (1986) establece las notas distintivas de una noción filosófica y antropológica de ambiente, luego reelaborada por Ingold. Esta refiere al involucramiento pragmático y material entre una persona y el entorno que lo rodea. No hay ambiente sin un organismo rodeado por elementos significativos. Personas, animales, organismos y entidades inorgánicas comparten las percepciones de lo que un ambiente ofrece o posibilita (a diferencia de teorías como la de Von Uexküll en la cual cada organismo se mantiene dentro de su Umwelt). ${ }^{13}$ La percepción no es una operación de constitución del objeto por parte de algún proceso cognitivo del sujeto sino un involucramiento directo del individuo dentro del ambiente. En esa inmersión hay un vínculo práctico con el entorno en el cual la persona atiende a ciertas propiedades significativas. Es decir que el vínculo primario con el entorno es más una acción exploratoria que una imposición

11 A este respecto puede resultar adecuado invitar a la reflexión a la noción de antropismo débil desarrollada en el escrito Más allá del principio antrópico. Hacia una filosofía del outside de Ezequiel Ludueña Romandini. Allí el autor destaca la preeminencia de cierta noción de vida en la crítica contemporánea al humanismo filosófico en la cual se conserva una concepción de lo humano como eslabón necesario en la cadena de la creación. De este modo, si bien el principio antropológico es rechazado tanto en la ciencia como en la filosofía, se conserva un principio antrópico débil a través de la persistencia de la vida como principio subrepticio de razonamiento. Véase Ludueña Romandini, E. (2012) Más allá del principio antrópico. Hacia una filosofía del outside. Buenos Aires: Prometeo.

12 Esta primera conclusión con respecto a la postura de Ingold puede contraponerse a la tesis de Jean Bennett en Vibrant Matter (2010), la cual también indaga en el problemático vínculo entre materia y vida. En este caso la problemática de la agencia también tiene un rol preponderante, aunque la propuesta de la autora con respecto a esta cuestión se ubicaría en la vereda contraria a la del antropólogo.

13 La contraposición entre las teorías de Gibson y Von Uexküll es desarrollada por Ingold en su escrito temprano Culture and the perception of the environment incluido en: Croll, E. y Parkin, D. (1992) Bush Base, Forest Farm. Culture, Environment, and Development. London: Routledge. 
de categorías. Este contacto directo con el ambiente se manifiesta en términos de lo que Gibson denomina affordances (posibilidades de acción). ${ }^{14}$ Percibir una affordance no es clasificar un objeto, sino que es un conocimiento que el organismo extrae por percepción directa del ambiente en el cual está involucrado. El existente humano está inmerso en este tipo de relación la mayor parte del tiempo. Es decir, se habla de ambiente cuando nos adentramos en el terreno del involucramiento material, en cambio, cuando se establece el distanciamiento requerido por el pensamiento clasificatorio y categorial, hablamos en términos de naturaleza. En palabras de Ingold:

El ambiente, como distinto de la naturaleza del mundo físico, es la misma realidad constituida en su relación con un sujeto, o grupo de sujetos, en su activo involucramiento con él. No es conocido por separado, previo a la acción, es por la acción en el mundo que las personas lo conocen, y puede percibir lo que posibilita $(1992,48) .{ }^{15}$

En esta concepción del entorno como posibilitador de acciones se evidencia un modo de contrarrestar el pensamiento por el cual la materia se conforma como un polo pasivo e inerte a la espera de una determinación. El vínculo entre las competencias del agente y las affordances del ambiente no puede pensarse en términos de activo/pasivo. La materia posee un orden y un significado propio que serán descubiertos y aprovechados en distinta medida por las competencias de los distintos organismos y puesto en relación con diferentes características y particularidades en cada caso. Será el involucramiento material el que determina el tipo de vínculo que se establecerá.

Ahora bien, ¿Cómo entendemos en este marco la idea de involucramiento material? Como objetivo principal, Ingold apunta a desactivar el binomio que separa lo material de lo inmaterial, el cual es solidario con la separación entre mente y materia, dualismo que está a la base de las nociones de agencia e intencionalidad. No hay nada que sea inmaterial, al contrario, existen materiales conectados por distintos tipos de superficies. Siguiendo nuevamente a Gibson (1986), debemos entender el ambiente como conformado por tres elementos: Medio, substancias y superficies. El medio es aquello que permite el movimiento y la percepción en general, para los humanos el medio por excelencia es el aire. Las substancias son relativamente resistentes al movimiento. Son elementos más o menos sólidos que nos permiten sostenernos. Las superficies son la interfase que separa y a su vez vincula los medios a las substancias. Ingold afirma: "Es por las superficies (surfaces) que los objetos son revelados a la percepción. Toda superficie es una interfase entre una sustancia más o menos sólida de un objeto y un medio volátil que lo rodea" $(2010,6) .{ }^{16}$ Ahora bien, lo que las superficies separan es un tipo de material de otro, no a lo material de lo inmaterial. Tanto las sustancias como el medio forman parte de los materiales.

Del mismo modo que la noción de ambiente se contrapone a la de naturaleza, la noción de materiales debe contraponerse a la de materialidad. Mediante este último concepto Ingold apunta a la reflexión metafísica solidaria con la división mente/materia que se inte-

14 Adoptaremos esta traducción por ser la más aceptada entre los investigadores que han escrito sobre el tema y la que de mejor manera refleja el objetivo que persigue Gibson con este neologismo.

15 La traducción es mía.

16 La traducción es mía. 
rroga acerca de aquello que hace que las cosas tengan cosidad, antes que hacer foco en los materiales a partir de los cuales están hechas y a los procesos a los cuales están supeditados. La materialidad apunta a ver en el entorno objetos cristalizados más que a materiales en movimiento, un mundo muerto, más que uno vivo en constante generación y transformación. En los estudios de cultura material existe un constante desliz desde los materiales hacia la materialidad. Ingold se pregunta:

¿Qué clase de perversión académica nos lleva a referirnos no a los materiales y sus propiedades sino a la materialidad de los objetos? Creo que el concepto de materialidad, sea cual fuere su significado, se ha convertido en un obstáculo real para la coherente investigación de los materiales, sus transformaciones y potencialidades [affordances]. $(2013,21)$.

La materialidad pretende pensar en torno a la materia, pero no parte de ella sino que replica los dualismos metafísicos de los cuales reniega. Sin aceptarlo, su punto de partida es fuertemente antropocéntrico. La curiosidad por la "materialidad" no tiene, según el autor, su motivación en la materia misma, sino que responde a un presupuesto de orden conceptual. Por el contrario, el investigador que parte de los materiales no puede tomar como punto de partida otra cosa que no sea el total involucramiento en ellos. La búsqueda de la "materialidad de los objetos" requiere necesariamente de un distanciamiento, una mirada natural. La operación por la cual nos distanciamos y observamos nuestro entorno como un contenedor de objetos nunca puede ser una vía fructífera para superar los dualismos antedichos. En lugar de una mente que informa la materia nos encontramos ante un ambiente en el cual las formas se generan por la acción de los materiales que se mezclan y se fusionan. No hay un adentro y un afuera de la materia, hay superficies de todo tipo que separan y conectan diferentes tipos de materiales. El existente humano es parte integrante de un océano de materiales en el cual está inmerso. En "Los materiales contra la materialidad" el autor lo explica de este modo:

Como cualquier otra criatura, los seres humanos no existen al "otro lado" de la materialidad sino que nadamos en un océano de materiales. Una vez que nos damos cuenta de nuestra inmersión, lo que este océano nos revela no es la homogeneidad anodina de los distintos matices de la materia, sino un flujo en el que materiales de los más diversos tipos - a través de procesos de adición y destilación, de coagulación y dispersión, y de evaporación y precipitación - sufren una continua generación y transformación. Las formas de las cosas, lejos de haber sido impuestas desde afuera sobre un substrato inerte, se elevan y son sostenidas - como también lo somos nosotros - dentro de esta corriente de materiales. Como con la misma Tierra, la superficie de cada sólido no es sino una corteza, el más o menos efímero congelamiento de un movimiento generativo $(2013,27)$.

Las propiedades de los materiales, que se nos manifiestan en sus superficies no son esencias sino momentos, o en palabras de Ingold, historias. Un mundo en que los objetos son cristalizaciones temporales resultantes de los flujos de la materia parece ofrecernos un panorama 
mucho más provechoso para contrarrestar la interpretación de la materia como un polo pasivo e inerte. Dicha contienda evita los ingredientes humanistas problemáticos que trae aparejada la introducción de la noción de agencia. A su vez, redimensiona el problema del vínculo humanono humano librándolo de los binomios que lo ataban a una disputa entre lo activo y lo pasivo.

\section{Conclusiones}

La cuestión de la agencia material, aunque motivada por propósitos fuertemente antihumanistas, encuentra dificultades muy complejas que ponen en duda su aporte a la reflexión posthumanista. Renovando la aporía expresada más arriba: o bien sostenemos el dualismo mente /materia, al cual el tópico de la agencia se adecúa de manera perfecta, o bien, adoptamos una postura fuertemente materialista que tome como punto de partida a los materiales cuestionando cualquier presupuesto, y tomando el riesgo de abandonar ciertas discusiones imposibles de resolver de manera cabal.

Por cuestiones de extensión, la profundización en las consecuencias políticas de este modo de entender la agencia material y su vínculo con otros autores que armonicen con esta concepción formarán parte de un próximo artículo. Entendemos que dicha discusión debería poner en diálogo lo analizado hasta aquí con autores como Karen Barad, Jane Bennett o Manuel de Landa. A este respecto, podemos mencionar que el hecho de considerar al existente humano como parte de un entramado de materiales, como propone Ingold, armoniza con tratamientos con respecto a la materia basados en intra-acciones (Barad). Esta noción remite a un modo de entender los fenómenos no como consecuencias de la interacción de entidades individuales sino como emergentes o resultados de intra-acciones materiales ${ }^{17}$. A partir de estas últimas emergen agencias ensambladas y dinámicas, con consecuencias políticas, gnoseológicas y estéticas acordes a esta naturaleza. Este modo de encarar la discusión pone el acento en evidenciar la politicidad intrínseca que emerge del movimiento de los materiales, problematizando el vínculo tradicional entre agencia y política signado por el materialismo dialéctico y el posestructuralismo.

Por lo pronto, el presente trabajo intentó evidenciar las dificultades que acarrea la búsqueda de una argumentación fuerte a favor de una simetría ontológica con respecto a la agencia. En el intento de democratizar la capacidad agentiva, se nos cuela por la ventana un motivo humanista arquetípico que remite directamente al humano como único motor de la reproducción de las formas. A su vez, se propone la necesidad de un cambio de enfoque a partir del cual pueda sostenerse una posición que se inscriba dentro del pensamiento materialista y posthumano pero conservando una noción de agencia que no se vea forzada a satisfacer necesariamente una simetría. Esto no complacerá, por supuesto, a las posturas posthumanistas más exigentes, ni a las pretensiones de sostener una distinción jerárquica fuerte, sin embargo, abonará a una concepción que redimensione la ubicación del existente humano en el entramado de materiales del cual forma parte. No creemos que una simetría con respecto a la agencia sea necesaria para sostener una postura posthumanista. Aun aceptando la imposibilidad de eliminar una asimetría de base entre la agencia humana y agencia material, puede defenderse una concepción posthumanista y materialista que aporte a una filosofía postantropocéntrica.

17 Acerca de esta noción véase Barad, K. (2007), Meeting the Universe Halfway: Quantum Physics and the Entanglement of Matter and Meaning, Durham y Londres: Duke University Press. 


\section{Referencias}

Bennett, J. (2010), Vibrant Matter, Carolina del Norte: Duke University Press.

Barad, K. (2007), Meeting the Universe Halfway: Quantum Physics and the Entanglement of Matter and Meaning, Durham y Londres: Duke University Press.

Coccia, E. (2017), La vida de las plantas, Buenos Aires: Miño y Dávila.

Gibson, J. J. (1986), The ecological approach to visual perception, New Jersey: Lawrence Elbaum Associates.

Ingold, T. (1992), "Culture and the perception of the environment", en: Croll, E. y Parkin, D. (eds.): Bush base: forest farm. Culture, environment and development, London: Routledge, pp. 39-56.

Ingold, T. (2000), The perception of the environment. Essays on livelihood, dwelling and skill, New York: Routledge.

Ingold, T. (2010), "Bringing Things to Life: Creative Entanglements in a World of Materials", Realities, Working Paper, 15, pp. 2-14.

Ingold, T. (2013), "Los materiales contra la materialidad", Papeles de trabajo, 11, pp. 19-39.

Latour, B. (1994), "On technical mediation. Philosophy, sociology, genealogy", Common knowledge, 3, pp. 29-64.

Latour, B. (1993), Nunca fuimos modernos, Madrid: Debate.

Ludueña Romandini, E. (2012), Más allá del principio antrópico. Hacia una filosofía del outside, Buenos Aires: Prometeo.

Malafouris, L. (2008), “At the Potter's Wheel: An Argument for Material Agency”, en: Knappett, C. y Malafouris, L., Material Agency. Towards a Non-anthropocentric Approach, New York: Springer, pp. 19-36.

Malafouris, L. (2013), How things shape the mind. A theory of material engagement, Massachussets: MIT Press.

Parente, D. (2016), "Los artefactos en cuanto posibilitadores de acción. Problemas en torno a la noción de agencia material en el debate contemporáneo", Rev. Colomb. Filos. Cienc. 16 (33), pp. 139-168.

Saerle, J. R., (1983), Intentionality: An Essay in the Philosophy of Mind, Cambridge: Cambridge University Press.

Sterelny, K. (2010), “Minds: extended or scaffolded?", Phenomenology and the Cognitive Sciences, 9 (4), pp. 465-481. 